\title{
Cell-based drug delivery harnesses inflammatory and autoimmune responses in neurodegeneration
}

\author{
David Pozo ${ }^{1,2}$ (D) \\ Received: 28 January 2021 / Revised: 22 February 2021 / Accepted: 25 February 2021 / Published online: 8 March 2021 \\ (C) Springer-Verlag GmbH Germany, part of Springer Nature 2021
}

Vaccine development, such a popular term in these pandemic times, is perhaps the most elegant example of how to co-opt an entire cellular and molecular machinery that has been finely tuned after millions of years of biological evolution for our own benefit. This is the ultimate rationale behind the biologyinspired, cell-based drug delivery systems that may overcome current approaches in drug design, even the more complex multitasks one, such as nanoparticles, cell ghosts or red blood cells.

In the Journal of Molecular Medicine [1], Levy, Rothhammer and Mascanfroni from the Quintana and the Karp laboratories at the Harvard Medical School in collaboration with researches at the King Abdullah International Medical Research Center in Riyadh take advantage of this bioinspired approach to rewire the behaviour of human mesenchymal stem cells (MSCs) to modify the disease course in a relapsingremitting multiple sclerosis (RRMS) murine model. Although MSCs might migrate to pathological areas - being this particularly relevant for tumour and hypoxic microenvironments - a major challenge to their therapeutic potential and utilization is that homing of MSCs is somewhat limited when they are systematically infused [2]. In this sense, the authors used MSCs as a Trojan horse by loading them with the staurosporine analog and protein kinase $\mathrm{C}$ inhibitor Ro-31-8425 with a double purpose (Fig. 1). First, they exploit the ability of Ro-31-8425 to modulate inflammatory responses and to enhance gene and

David Pozo

david.pozo@cabimer.es; dpozo@us.es

1 Cellular and Molecular Neuroimmunology Laboratory, CABIMER, Andalusian Centre for Molecular Biology and Regenerative Medicine, CSIC-UPO-University of Seville, CABIMER Bldg. Av. Americo Vespucio, 24, Seville, Spain

2 Department of Medical Biochemistry, Molecular Biology and Immunology, University of Seville Medical School, Seville, Spain surface expression in MSCs of the integrin CD11a [3], a key ligand for ICAM-1-mediated adhesion and homing towards activated endothelial cells. Second, the strategy of using MSCs as a systemic cell-based delivery tool allows a steady release into blood and a considerable increase in circulating concentrations compared to the free drug. Surprisingly, drugloaded human MSC did not change their tissue distribution but had a differential impact on the immune responses in the experimental autoimmune encephalomyelitis (EAE) model of multiple sclerosis (MS) compared to free-drug treatments. This study is the first to report an improved prophylactic effect of human MSCs used as a cell-based drug delivery system in a disease model with both inflammatory and autoimmune components. MSC-delivered Ro-31-8425 decreases the brain CD4+IL17+ cells and proinflammatory macrophages recruitment which led to a significant amelioration of the clinical score in the MS murine model. Remarkably, these effects are greater compared to those obtained only with human MSCs or Ro-31-8425. Although the molecular mechanisms are not completely understood (CD11a is crucial for the optimal expansion of antigen-specific CD8 and CD4 T cells, alterations in the MSCs secretome, differential effects on protein kinases on immunocompetent cells, etc.), these results are highly encouraging for further exploration of the pleiotropic effects emerging from cell-based delivery systems in order to generate biohybrid drugs. 
Fig. 1 Human mesenchymal stem cells as a biohybrid drug in multiple sclerosis

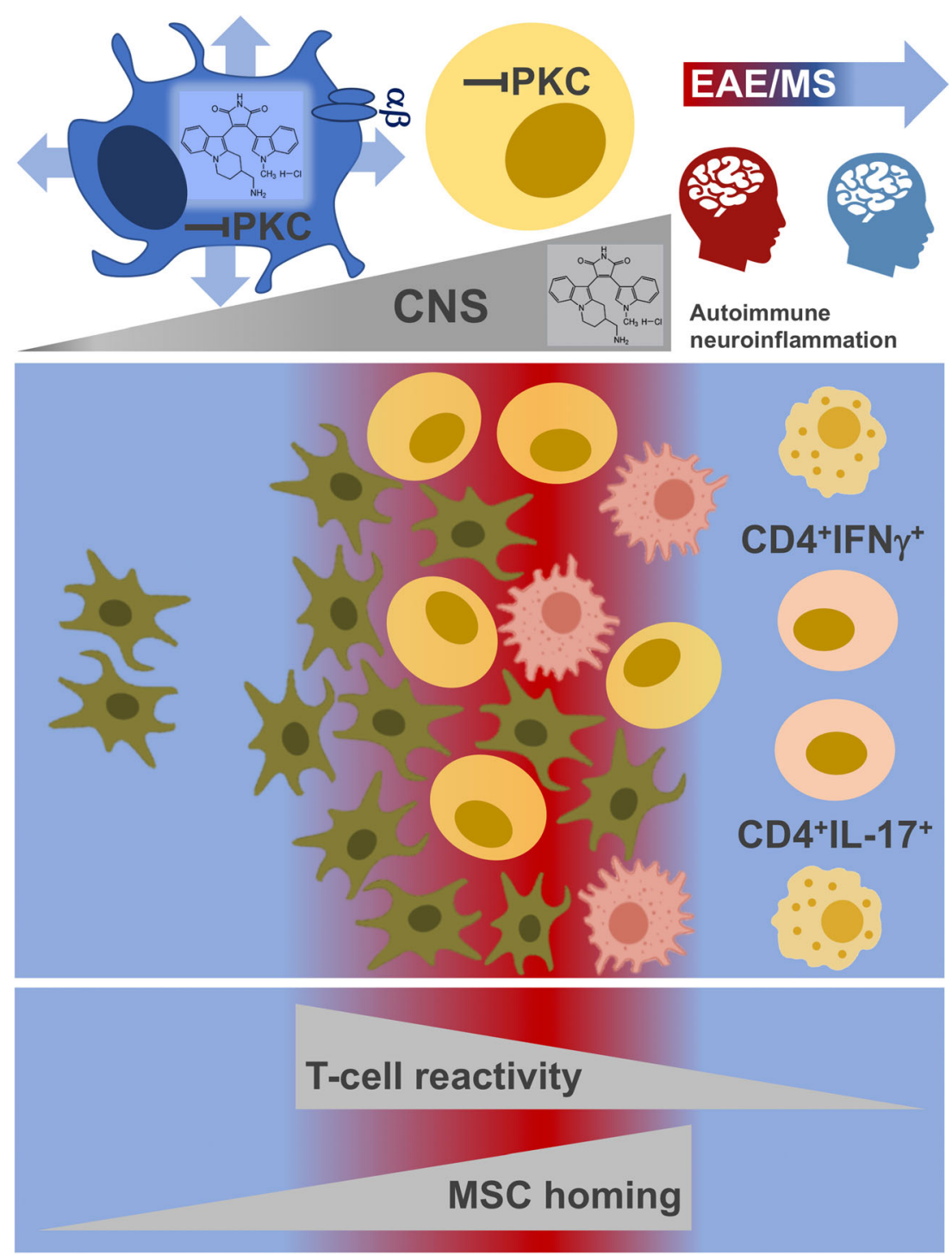

\section{Declarations}

Conflict of interest The author declares no competing interests.

\section{References}

1. Levy O, Rothhammer V, Mascanfroni I, Tong Z, Kuai R, de Biasio M, Wang Q, Majid T, Perrault C, Yeste A, Kenison JE, Safaee H, Musabeyezu J, Heinelt M, Milton Y, Kuang H, Lan H, Siders W, Multon MC, Rothblatt J, Massadeh S, Alaamery M, Alhasan AH, Quintana FJ, Karp JM (2021) A cell-based drug delivery platform for treating central nervous system inflammation. J Mol Med. https://doi. org/10.1007/s00109-020-02003-9

2. Zenke M (2017) Stem cells: from biomedical research towards clinical applications. Journal of Molecular Medicine 95:683-685

3. Levy O, Mortensen LJ, Boquet G, Tong Z, Perrault C, Benhamou B, Zhang J, Stratton T, Han E, Safaee H, Musabeyezu J, Yang Z, Multon MC, Rothblatt J, Deleuze JF, Lin CP, Karp JM (2015) A small-molecule screen for enhanced homing of systemically infused cells. Cell Rep. 10:1261-1268

Publisher's note Springer Nature remains neutral with regard to jurisdictional claims in published maps and institutional affiliations. 